\title{
riccafd
}

Revista Iberoamericana de Ciencias de la Actividad Física y el Deporte

\section{FATIGA OCASIONADA POR DIFERENTES FORMATOS DE JUEGOS REDUCIDOS EN FUTBOLISTAS JÓVENES}

\section{RELATED-FATIGUE BY DIFFERENT SMALL-SIDED GAMES FORMATS IN YOUNG SOCCER PLAYERS}

\author{
Castillo, D. ${ }^{1}$, Domínguez-Díez, M. ${ }^{1}$, Flores-López, M. ${ }^{1}$, Raya-González, J. ${ }^{1}$ \\ ${ }^{1}$ Castillo, D. \\ Facultad de Ciencias de la Salud, Universidad Isabel I de Castilla, Burgos, España. \\ ${ }^{1}$ Domínguez-Díez, M. \\ Facultad de Ciencias de la Salud, Universidad Isabel I de Castilla, Burgos, España. \\ ${ }^{1}$ Flores-López, M. \\ Facultad de Ciencias de la Salud, Universidad Isabel I de Castilla, Burgos, España. \\ ${ }^{1}$ Raya-González, J. \\ Facultad de Ciencias de la Salud, Universidad Isabel I de Castilla, Burgos, España.
}

Correspondencia: Raya-González, J.javier.raya@ui1.es

Código UNESCO: 5801 Teoría y métodos educativos

Clasificación Consejo de Europa: 12. Aprendizaje Motor

Recibido el 23 de octubre de 2018

Aceptado el 26 de febrero de 2019

DOI: http://dx.doi.org/10.24310/riccafd.2019.v8i2.6455

\section{RESUMEN}

El principal objetivo de este estudio fue analizar la influencia de diferentes formatos de juego reducido (JR) en la carga interna medida mediante el esfuerzo percibido ( $\mathrm{Cl} \mathrm{EP}$ ) declarado por los futbolistas, y en el rendimiento del salto horizontal $(\mathrm{SH})$ y la capacidad de acelerar y esprintar. Diez futbolistas cadetes (sub-16) participaron en el estudio. Los jugadores disputaron 4 formatos de JR con modificaciones en el espacio de juego y en la duración de las repeticiones. Los participantes realizaron un esprint de $30 \mathrm{~m}$ y un $\mathrm{SH}$ antes y después de cada repetición y también declararon el EP. Los resultados mostraron una mayor $\mathrm{Cl}$ EP. Además, se observó un empeoramiento del esprint en 10 y 30 m después de la última repetición. Este conocimiento podría ayudar a los cuerpos técnicos a establecer de manera más apropiada la duración total y de cada repetición de los JRs.

Palabras vlave: fútbol, cuantificación, esfuerzo percibido, fatiga, rendimiento neuromuscular. 


\section{ABSTRACT}

The main aim of this study was to analyze the influence of different small-sided games (SSGs) on the internal load measured by the rating of perceived exertion (sRPE) declared by the soccer players and on the horizontal jump (HJ) and acceleration and sprint performances. Ten U16 soccer players participated in this study. The players played 4 SSGs formats with modifications in the pitch size and in the bouts duration. The participants performed a $30 \mathrm{~m}$ sprint and a $\mathrm{HJ}$ before and after each bout and they declared the RPE. The results showed a higher SRPE. In addition, a worsening sprint performance at 10 and $30 \mathrm{~m}$ after the last bout was observed. This knowledge could help the technical staff to establish more appropriately the total and bout duration of the SSGs.

Key words: soccer; quantification; perceived exertion; fatigue; neuromuscular performance.

\section{INTRODUCCIÓN}

La utilización de juegos reducidos (JRs) en los entrenamientos es una estrategia comúnmente utilizada por los cuerpos técnicos ya que tienen una estructura muy similar al juego real a pesar de ser jugados en espacios menores $(1,2)$. Estas tareas de entrenamiento de colaboración-oposición, participación simultánea, en espacio compartido y con una clara orientación del juego permiten el desarrollo de las habilidades técnico-tácticas por parte de los jugadores así como la mejora de aspectos condicionales específicos del fútbol $(3,4)$. En función de las necesidades de los cuerpos técnicos y atendiendo al objetivo perseguido, los JRs pueden ser disputados en formatos muy diferentes, sobre todo, gracias a la modificación de algunas variables tales como el espacio de juego, la duración de las repeticiones, la duración de la recuperación, la orientación del espacio, el número de toques, la introducción de reglas, el tipo de marcaje, el objetivo final o el aliento del entrenador (5-9). Así pues, el hecho de modificar estas variables puede afectar tanto a las respuestas físicas y fisiológicas de los jugadores (10) como al esfuerzo percibido (EP) declarado por los futbolistas (11), factores muy relevantes a tener en cuenta en el diseño y aplicación de los JRs durante los entrenamientos semanales.

La influencia de distintos formatos de JRs en el EP declarado por los futbolistas ha sido poco estudiada en la literatura (12-14). Concretamente, respecto al espacio de juego, Casamichana y Castellano (12) constataron que a medida que el espacio individual de interacción (EII) fue incrementado (p.e., pequeño = $75 \mathrm{~m}^{2}$, mediano $=175 \mathrm{~m}^{2}$ y grande $=275 \mathrm{~m}^{2}$ ), los jugadores declaraban un EP mayor. En cambio, otros autores, a pesar de observar similares diferencias entre los JRs disputados en espacio pequeño $\left(E I I=56 \mathrm{~m}^{2}\right)$ respecto a espacio mediano $\left(E I I=87 \mathrm{~m}^{2}\right)$ y grande $\left(E I I=126 \mathrm{~m}^{2}\right)$, no constataron diferencias en el EP declarado por los jugadores en los JRs disputados en Ell medianos y grandes (13). Parece que no existe un consenso en la literatura acerca de la nomenclatura en cuanto a las dimensiones del espacio de juego en los JRs, siendo pre- 
ciso utilizar el término Ell para hacer referencia a los distintos formatos de JR. Por otro lado, atendiendo a la duración de los JR, Fanchini et al. (14) observaron un aumento del EP declarado por los futbolistas en las últimas repeticiones de los JR no siendo esta variable influenciada por la duración de las mismas (p.e., 4 y 6 min). Estas investigaciones fueron llevadas a cabo con futbolistas sénior, sin embargo, debido al escaso conocimiento sobre la influencia de las distintas modificaciones en los JR sobre el EP en categorías inferiores de fútbol y que se trata de un método válido, barato y fácil de utilizar (15), sería interesante profundizar en el análisis de las posibles diferencias existentes en el EP atendiendo al espacio de juego, a la duración de las series y a la combinación de ambos factores en el diseño de los JRs.

Para determinar la fatiga producida por los distintos formatos de JRs no es suficiente con realizar un análisis de las respuestas objetivas y/o subjetivas registradas por los jugadores a lo largo de la disputa de los mismos, sino que también se necesita de la realización de pruebas de rendimiento físico realizadas antes y después de cada JR (16). En este sentido, algunos autores han mostrado una pérdida de rendimiento en la capacidad de esprintar en 5 y $15 \mathrm{~m}$ en un JR disputado en una situación de 4 vs 4 (con porteros) (17). En cambio, Muñoz, Castillo y Yanci (18), en un JR de 5 vs 5 (con porteros) no observaron tales diferencias en distancias de $15 \mathrm{~m}$. Así mismo, otras investigaciones han analizado el efecto de los JR en la capacidad de salto vertical obteniendo resultados totalmente contradictorios. Mientras algunos autores observaron un descenso en la capacidad de salto vertical tras la disputa de un JR (19), otros autores no constataron tales pérdidas (20). A pesar de que se ha analizado la fatiga inducida por distintos formatos de JRs en la capacidad de aceleración y salto vertical, no se conocen trabajos que hayan analizado la pérdida de rendimiento en otras habilidades relevantes para el rendimiento en fútbol como la capacidad de salto horizontal (SH) $(21,22)$.

Por último, dado que no se han encontrado estudios que hayan analizado la fatiga neuromuscular, entendida como la pérdida de rendimiento físico, después de cada repetición de diferentes formatos de JRs, proporcionar este conocimiento sería de gran utilidad para los cuerpos técnicos ya que permitiría comprender los efectos que tienen los JRs en sus jugadores y poder así planificar de forma más rigurosa los contenidos semanales de entrenamiento, así como establecer de forma más adecuada la duración y descanso de las repeticiones de los JRs. Por lo tanto, el principal objetivo de este estudio fue analizar la influencia de diferentes formatos de juego reducido (JR) en la carga interna medida mediante el esfuerzo percibido (Cl EP) declarado por los futbolistas y en el rendimiento del salto horizontal $(\mathrm{SH})$ y la capacidad de acelerar y esprintar.

\section{MATERIAL Y MÉTODOS}

\section{Participantes}

En este estudio participaron un total de 10 futbolistas (edad $=14,8 \pm 0,6$ años, estatura $=173 \pm 7 \mathrm{~cm}$, masa $=60,6 \pm 8,1 \mathrm{~kg}$, años de experiencia en el $\mathrm{Club}=3,8$ 
$\pm 2,6$ años) pertenecientes a la categoría cadete (sub-16). Los jugadores entrenaban 3 días a la semana y disputaban un partido oficial los fines de semana. El criterio de inclusión para formar parte del estudio fue no haber estado lesionado en el momento durante el mes previo a la intervención y participar en los 4 formatos de JRs propuestos. Entrenadores, jugadores y padres/madres o tutores legales fueron informados del procedimiento de investigación, los requerimientos y los riesgos potenciales previamente a firmar el consentimiento informado. El estudio siguió las pautas marcadas en la Declaración de Helsinki (2013), así como los estándares éticos para la investigación del ejercicio y del deporte con seres humanos (23).

\section{Diseño experimental}

El estudio se llevó a cabo durante el mes de febrero de la temporada 20172018. Los jugadores participaron en 4 formatos de JRs durante 4 entrenamientos con al menos 72 horas de recuperación entre cada uno y con al menos $48 \mathrm{~h}$ de recuperación desde el último partido. Los futbolistas fueron instruidos para realizar su última comida tres horas antes del comienzo de las pruebas, no tomar bebidas con cafeína, ni realizar ejercicio físico intenso el día de la toma de datos (24). Todos los JR fueron disputados a la misma hora (20:00) y en el mismo campo de hierba artificial de última generación. Antes de comenzar los JR, se realizó un calentamiento estandarizado que consistía en 7 min de carrera continua y movilidad articular, 10 min de posesiones en espacio reducido y 3 esprines progresivos y aceleraciones. Los jugadores realizaron un esprint de $30 \mathrm{~m}$ y un SH antes y después de cada repetición de JR. Además, al terminar cada repetición los jugadores declararon el EP (Figura 1). Durante el desarrollo de los diferentes JRs así como en la realización de los distintos test los deportistas utilizaron sus botas de fútbol habituales. Los investigadores motivaron y animaron a los participantes para que realizaran estas pruebas con la mayor intensidad posible.

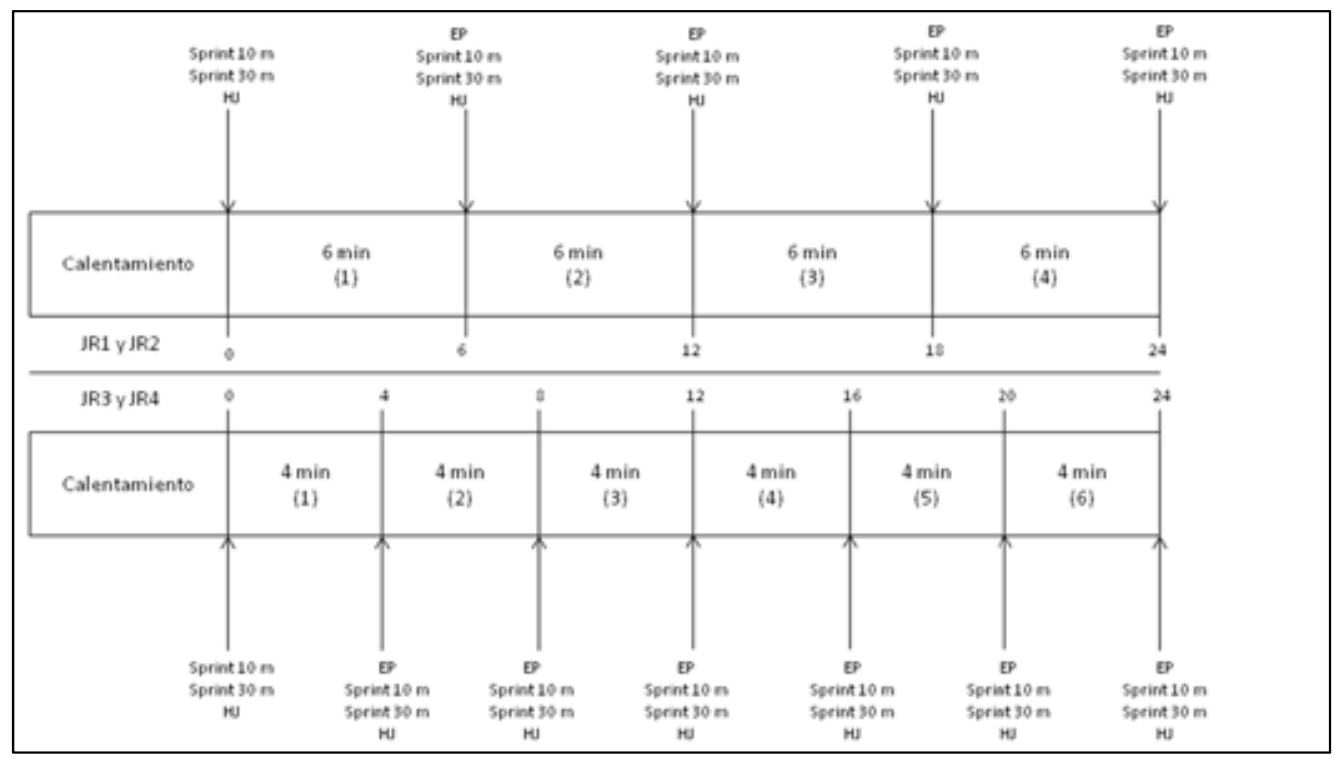

Figura 1. Secuencia temporal de la recogida de datos en los distintos juegos reducidos (JRs). 
Juegos reducidos (JRs): Se disputaron 4 formatos de JRs con modificaciones en el espacio de juego y en el tiempo de descanso entre las repeticiones (Tabla 1). En todos los JRs se enfrentaban 2 equipos compuestos por 6 jugadores (portero incluido) cada uno. En todo momento los jugadores contaron con la presencia y aliento del entrenador y se reemplazaba el balón cada vez que salía fuera de los límites del terreno de juego para dotar a los JRs de un mayor tiempo de juego efectivo (25).

Tabla 1. Formato de los juegos reducidos (JR).

\begin{tabular}{cccc}
\hline JR & $\begin{array}{c}\text { Repeticiones } \times \text { duración } \\
(\mathrm{min})\end{array}$ & $\begin{array}{c}\text { Dimensiones } \\
(\mathrm{m})\end{array}$ & $\begin{array}{c}\text { EII } \\
\left(\mathrm{m}^{2}\right)\end{array}$ \\
\hline JR1 & $4 \times 6$ & $38 \times 26$ & 100 \\
JR2 & $4 \times 6$ & $53 \times 37$ & 200 \\
JR3 & $6 \times 4$ & $38 \times 26$ & 100 \\
JR4 & $6 \times 4$ & $53 \times 37$ & 200 \\
\hline
\end{tabular}

EII = espacio individual de interacción.

Esprint 10 y $\mathbf{3 0}$ m: Los participantes completaron un esprint en línea recta en una distancia de $30 \mathrm{~m}$ (16). La salida se realizó a $0,5 \mathrm{~m}$ de la primera de las tres fotocélulas empleadas (Microgate ${ }^{\circledR}$ Polifemo, Bolzano, Italia). El tiempo (s) registrado a los $10 \mathrm{~m}$ (primera fotocélula, esprint $10 \mathrm{~m}$ ) y a los $30 \mathrm{~m}$ (segunda fotocélula, esprint $30 \mathrm{~m}$ ) fue registrado. El software Witty Manager (Microgate ${ }^{\circledR}$ Polifemo, Bolzano, Italia) fue utilizado para descargar y gestionar los datos relativo a los diferentes esprines.

Salto horizontal (SH): Los participantes ejecutaron dos $\mathrm{SH}$ bilaterales con contramovimiento y brazos libres. Para la realización de los saltos, los jugadores se colocaban detrás de una línea y tras la flexión de rodillas, llevaron a cabo el salto en la horizontal, marcando como distancia de salto la distancia entre la línea desde la que se iniciaba el salto y el punto de apoyo del talón más próximo a esta línea (26). Para medir la distancia del salto se utilizó una cinta métrica colocada en el suelo. El mejor salto fue utilizado para los análisis posteriores.

Esfuerzo percibido (EP): La carga interna medida mediante el EP (CI EP) fue registrada mediante el método utilizado por Foster et al. (27). Inmediatamente tras la finalización de cada repetición de JR a cada futbolista se le mostraba la escala RPE de 0-10 (27) y el jugador declaraba un valor que posteriormente se multiplicaba por la duración de cada repetición (p.e., 4 o 6 min). Los jugadores respondieron a la pregunta - ¿cómo de duro ha sido el juego reducido? - realizada siempre por la misma persona (p.e., investigador principal). Los jugadores declararon el EP de manera sistemática atendiendo al orden de realización de los tests físicos, sin la presencia de otros compañeros y teniendo restringido el acceso a los valores declarados por los compañeros (15). Los 
jugadores se familiarizaron con el uso de la escala de EP durante el mes previo a la investigación.

\section{Análisis estadístico}

Los resultados se presentan como media \pm desviación típica (DT). Para calcular las diferencias en el rendimiento de $\mathrm{SH}$ y esprint así como la $\mathrm{Cl}$ EP antes y después de cada repetición en cada uno de los JRs se utilizó el método de inferencia propuesto por Hopkins, Marshall, Batterham y Hanin (28) basado en el cálculo de las magnitudes de las diferencias $(<0,2$ baja; 0,2-0,6 moderada, 0,61,2 alta; 1,2-2,0 muy alta; >2,0 extremadamente alta). Además, se calculó el $90 \%$ del límite de confianza ( $\pm 90 \%$ LC) y la probabilidad de que las diferencias fueran ciertas atendiendo a los siguientes rangos: $25-75 \%$, posible $75-95 \%$, probable; 95-99,5\%, muy probable; $>99,5 \%$, extremadamente probable (28). Por otro lado, se calculó la asociación entre el porcentaje de cambio en cada test de rendimiento (p.e., SH, esprint $30 \mathrm{~m}$, esprint $10 \mathrm{~m}$ ) y la $\mathrm{Cl}$ EP total acumulada durante las distintas repeticiones de cada JR mediante la correlación de Pearson ( $r$ ). Para la interpretación de las magnitudes de las correlaciones se utilizó la siguiente escala: menor que 0,1, trivial; de 0,1 a 0,3, baja; de 0,3 a 0,5, moderada; de 0,5 hasta 0,7 , alta; 0,7-0,9, muy alta; mayor que 0,9, casi perfecta (28). Además se calculó el $\pm 90 \%$ LC y las probabilidades de que las asociaciones fueran ciertas. El análisis estadístico se realizó con el programa Statistical Package for Social Sciences (SPSS Inc, versión 23,0 Chicago, IL, EE.UU.).

\section{RESULTADOS}

En la tabla 2 se presenta la CI EP registrada por los futbolistas después de cada repetición de los distintos JRs y la CI total acumulada. La última repetición de todos los JRs supone una CI EP mayor (muy probable - extremadamente probable grande) en comparación de la primera.

Tabla 2. Resultados de la carga interna medida mediante esfuerzo percibido (CI EP) declarado por los futbolistas después de cada repetición de los distintos formatos de juegos reducidos (JR) y la carga total acumulada.

\begin{tabular}{cccccccccc}
\hline & JR & 1 & 2 & 3 & 4 & 5 & 6 & Cl EP total & $\Delta \%$ \\
\hline & JR1 & $27,6 \pm 5,1$ & $34,2 \pm 7,5$ & $37,8 \pm 8,0$ & $39,0 \pm 9,1$ & - & - & $138,6 \pm 23,4$ & $11,4 \pm 5,8$ \\
& JR2 & $24,0 \pm 4,0$ & $30,6 \pm 7,2$ & $35,4 \pm 7,2$ & $38,4 \pm 9,0$ & - & - & $128,4 \pm 23,0$ & $14,4 \pm 5,5$ \\
CI EP & & & & & & & & \\
& JR3 & $15,6 \pm 4,4$ & $18,4 \pm 3,9$ & $19,2 \pm 3,7$ & $19,2 \pm 3,7$ & $19,6 \pm 3,0$ & $20,4 \pm 4,0$ & $112,4 \pm 20,0$ & $4,8 \pm 1,8$ \\
& JR4 & $15,6 \pm 3,5$ & $18,8 \pm 4,6$ & $20,4 \pm 6,4$ & $22,0 \pm 3,4$ & $21,6 \pm 3,9$ & $24,0 \pm 5,3$ & $122,4 \pm 21,9$ & $8,4 \pm 4,2$ \\
\hline
\end{tabular}


Tabla 3. Resultados en el salto horizontal $(\mathrm{SH})$ registrados por los futbolistas antes y después $(\Delta \%)$ de cada repetición de los distintos formatos de juegos reducidos $(J R)$

\begin{tabular}{|c|c|c|c|c|c|c|c|c|c|c|c|}
\hline Test & $J R$ & Pre & 1 & 2 & 3 & 4 & 5 & 6 & $\Delta \%$ & $\begin{array}{c}\text { TE } \\
(90 \% \mathrm{CL})\end{array}$ & $\begin{array}{l}\text { Inferencia } \\
\text { cualitativa }\end{array}$ \\
\hline \multirow{4}{*}{ SH } & JR1 & $213 \pm 17$ & $210 \pm 14$ & $203 \pm 12$ & $206 \pm 9$ & $204 \pm 14$ & - & - & $-0,9 \pm 6,3$ & $-0,53 \pm 0,36$ & $\begin{array}{l}\text { Probable } \\
\text { Pequeña } \\
(94 / 6 / 0)\end{array}$ \\
\hline & $J R 2$ & $202 \pm 12$ & $204 \pm 14$ & $205 \pm 14$ & $203 \pm 10$ & $202 \pm 14$ & $\cdot$ & - & $0,6 \pm 4,6$ & $0,04 \pm 0,33$ & $\begin{array}{l}\text { Poco clara } \\
\text { (20/69/11) }\end{array}$ \\
\hline & JR3 & $207 \pm 9$ & $213 \pm 14$ & $216 \pm 11$ & $213 \pm 13$ & $209 \pm 7$ & $210 \pm 10$ & $208 \pm 12$ & $1,5 \pm 6,3$ & $0,13 \pm 0,55$ & $\begin{array}{l}\text { Poco clara } \\
(41 / 44 / 15)\end{array}$ \\
\hline & JR4 & $212 \pm 12$ & $207 \pm 18$ & $214 \pm 11$ & $211 \pm 10$ & $212 \pm 13$ & $210 \pm 12$ & $213 \pm 12$ & $0,8 \pm 8,6$ & $0,06 \pm 0,68$ & $\begin{array}{l}\text { Poco clara } \\
(36 / 39 / 25)\end{array}$ \\
\hline
\end{tabular}

En la tabla 3 se muestran los resultados obtenidos por los jugadores en el rendimiento de SH Las diferencias encontradas fueron poco claras y probables pequeñas en el rendimiento de SH antes y después de la última repetición en los distintos JR.

En la tabla 4 se muestran los resultados obtenidos por los jugadores en el rendimiento de esprint en distancias de 10 y $30 \mathrm{~m}$. En todos los JRs aumentó el tiempo de forma muy probable - extremadamente probable pequeñas - moderadas - grandes en el rendimiento en el esprint en distancias de 10 y $30 \mathrm{~m}$ después de la última repetición respecto al rendimiento antes del comienzo de la primera repetición, excepto en el JR2 que se encontraron diferencias posible pequeñas.

Tabla 4. Resultados del rendimiento en el sprint de 10 y $30 \mathrm{~m}$ registrado por los futbolistas antes y después $(\Delta \%)$ de cada repetición de los distintos formatos de juegos reducidos (JR)

\begin{tabular}{|c|c|c|c|c|c|c|c|c|c|c|c|}
\hline & & Pre & 1 & 2 & 3 & 4 & 5 & 6 & $\Delta \%$ & $\begin{array}{c}\text { TE }(90 \% \\
\mathrm{CL})\end{array}$ & $\begin{array}{l}\text { Inferencia } \\
\text { cualitativa }\end{array}$ \\
\hline \multirow{4}{*}{$\begin{array}{l}10 \mathrm{~m} \\
\text { Sprint }\end{array}$} & JR1 & $1,85 \pm 0,07$ & $1,90 \pm 0,09$ & $1,94 \pm 0,10$ & $1,92 \pm 0,10$ & $1,95 \pm 0,09$ & $\cdot$ & - & $0,1 \pm 0,1$ & $1,14 \pm 0,65$ & \multirow{4}{*}{$\begin{array}{c}\text { Muy Probable } \\
\text { Grande } \\
\text { (99/1/0) } \\
\text { Posible Pequeña } \\
\text { (64/31/5) } \\
\text { Muy Probable } \\
\text { Pequeña }(97 / 2 / 1) \\
\text { Muy Probable } \\
\text { Moderada (98/2/10) }\end{array}$} \\
\hline & JR2 & $1,90 \pm 0,09$ & $1,96 \pm 0,12$ & $1,98 \pm 0,15$ & $1,94 \pm 0,10$ & $1,94 \pm 0,13$ & - & $\cdot$ & $0,0 \pm 0,1$ & $0,30 \pm 0,47$ & \\
\hline & JR3 & $1,87 \pm 0,07$ & $1,90 \pm 0,08$ & $1,91 \pm 0,10$ & $1,93 \pm 0,10$ & $1,94 \pm 0,08$ & $1,94 \pm 0,06$ & $1,92 \pm 0,08$ & $0,1 \pm 0,0$ & $0,54 \pm 0,28$ & \\
\hline & $\mathrm{JR} 4$ & $1,83 \pm 0,11$ & $1,87 \pm 0,10$ & $1,88 \pm 0,10$ & $1,85 \pm 0,09$ & $1,88 \pm 0,07$ & $1,89 \pm 0,10$ & $1,89 \pm 0,09$ & $0,1 \pm 0,0$ & $0,65 \pm 0,34$ & \\
\hline \multirow{4}{*}{$\begin{array}{l}30 \mathrm{~m} \\
\text { Sprint }\end{array}$} & JR1 & $4,46 \pm 0,13$ & $4,61 \pm 0,21$ & $4,64 \pm 0,23$ & $4,64 \pm 0,21$ & $4,74 \pm 0,22$ & - & - & 0,1 & $1,41 \pm 0,48$ & \multirow{4}{*}{$\begin{array}{c}\text { Extremadamente } \\
\text { Probable Grande } \\
(100 / 0 / 0) \\
\text { Muy Probable } \\
\text { Moderada }(99 / 1 / 0) \\
\text { Muy Probable } \\
\text { Moderada }(99 / 1 / 0) \\
\text { Muy Probable } \\
\text { Moderada }(99 / 1 / 0)\end{array}$} \\
\hline & JR2 & $4,58 \pm 0,16$ & $4,70 \pm 0,23$ & $4,73 \pm 0,20$ & $4,72 \pm 0,21$ & $4,79 \pm 0,23$ & - & - & $0,2 \pm 0,1$ & $0,95 \pm 0,51$ & \\
\hline & JR3 & $4,62 \pm 0,22$ & $4,67 \pm 0,25$ & $4,72 \pm 0,25$ & $4,65 \pm 0,22$ & $4,71 \pm 0,19$ & $4,77 \pm 0,23$ & $4,77 \pm 0,22$ & $0,2 \pm 0,1$ & $0,64 \pm 0,26$ & \\
\hline & JR4 & $4,58 \pm 0,20$ & $4,63 \pm 0,18$ & $4,67 \pm 0,21$ & $4,75 \pm 0,25$ & $4,77 \pm 0,20$ & $4,75 \pm 0,16$ & $4,74 \pm 0,21$ & $0,2 \pm 0.1$ & $0,70 \pm 0,32$ & \\
\hline
\end{tabular}

La tabla 5 muestra las asociaciones entre el porcentaje de cambio en cada test de rendimiento y la CI EP total registrada en cada JR. Todas las asociaciones reportadas entre el test de $\mathrm{SH}$ y el esprint en distancias de 10 y $30 \mathrm{~m}$ con la $\mathrm{Cl}$ EP total acumulada en los JRs fueron poco claras. 
Tabla 5. Asociación ( $r ; \pm 90 \%$ LC) entre el porcentaje de cambio en cada test de rendimiento y la carga interna medida mediante el esfuerzo percibido (CI EP) en cada juego reducido (JR).

\begin{tabular}{ccccc}
\hline JR & Test & $(\mathrm{r} ; \pm 90 \% \mathrm{LC})$ & Inferencia cualitativa & Ratio \\
\hline \multirow{3}{*}{ JR1 } & SH & $0,10 \pm 0,55$ & Poco clara & $50 / 20 / 30$ \\
& Sprint $10 \mathrm{~m}$ & $0,37 \pm 0,50$ & Poco clara & $78 / 13 / 9$ \\
& Sprint $30 \mathrm{~m}$ & $0,20 \pm 0,54$ & Poco clara & $61 / 18 / 21$ \\
\hline \multirow{3}{*}{ JR2 } & SH & $0,25 \pm 0,53$ & Poco clara & $66 / 17 / 17$ \\
& Sprint $10 \mathrm{~m}$ & $-0,32 \pm 0,51$ & Poco clara & $13 / 14 / 73$ \\
& Sprint 30 m & $-0,32 \pm 0,51$ & Poco clara & $13 / 14 / 73$ \\
\hline \multirow{3}{*}{ JR3 } & SH & $0,09 \pm 0,55$ & Poco clara & $49 / 20 / 31$ \\
& Sprint $10 \mathrm{~m}$ & $0,29 \pm 0,52$ & Poco clara & $70 / 15 / 15$ \\
& Sprint 30 m & $0,27 \pm 0,52$ & Poco clara & $68 / 16 / 16$ \\
\hline \multirow{2}{*}{ JR4 } & SH & $0,13 \pm 0,55$ & Poco clara & $53 / 20 / 27$ \\
& Sprint 10 m & $0,12 \pm 0,55$ & Poco clara & $52 / 20 / 28$ \\
& Sprint 30 m & $0,33 \pm 0,51$ & Poco clara & $74 / 14 / 12$ \\
\hline
\end{tabular}

$r$ = coeficiente de correlación; $L C$ = límite de confianza; $\mathrm{SH}$ = salto horizontal

\section{Discusión}

El principal objetivo de este estudio fue analizar la influencia de diferentes formatos de juego reducido (JR) en la carga interna medida mediante el esfuerzo percibido (Cl EP) declarado por los futbolistas y en el rendimiento en la capacidad de salto $(\mathrm{SH})$ y en la capacidad de acelerar y esprintar. Los principales resultados obtenidos en este estudio mostraron que en la última repetición de los JRs analizados los jugadores registraron una CI EP mayor (muy probable - extremadamente probable grande) que en la primera. Además, mientras que las diferencias encontradas fueron poco claras y probables pequeñas en el rendimiento de SH después de la última repetición en los distintos JRs respecto a la primera, en todos los JRs aumentó el tiempo de forma muy probable - extremadamente probable pequeñas - moderadas - grandes en el rendimiento en el esprint en distancias de 10 y 30 m después de la última repetición respecto al rendimiento registrado antes de la realización de los JRs. Por último, se observó que las asociaciones reportadas entre el test de $\mathrm{SH}$ y el esprint en distancias de 10 y 30 m con la CI EP total acumulada en los JRs fueron poco claras.

Cuantificar la CI EP tras la realización de JRs es un método barato, útil y fácil de utilizar para los cuerpos técnicos de las categorías inferiores de los equipos de fútbol (29). Algunos autores mostraron que los jugadores declaraban valores de $6,7 \pm 0,8,6,7 \pm 0,8$ y 5,7 $\pm 1,0$ UA en Ell de 75,175 y $275 \mathrm{~m}^{2}$, respectivamente (12). Otros autores también observaron valores similares $(6,2 \pm 0,8$ y 6,2 $\pm 0,6$ en Ell de 87 y $126 \mathrm{~m}^{2}$, respectivamente) (13). Sin embargo, en nuestro estudio se encontraron valores más bajos de EP ( 5,5 UA) en todos los formatos de 
JRs. Estas diferencias pueden ser debidas a la inclusión de la regla del fuera de juego en nuestra investigación de cara a asemejar los JRs al juego real, lo cual, implica una mejor estructuración de los jugadores sobre el terreno de juego $(3,4)$. Conocer la percepción subjetiva de los jugadores a lo largo de las distintas tareas de entrenamiento es interesante para poder planificar los entrenamientos semanales y ubicar las tareas en el microciclo de manera más eficaz $(30,31)$. En este sentido, en nuestro estudio se observó que los jugadores declaraban una mayor CI EP en la última repetición de los distintos formatos de JRs disputados respecto a la primera, independientemente de las dimensiones y duración de las repeticiones. En consonancia con nuestro estudio, Fanchini et al. (14) observaron que el EP declarado por los jugadores fue mayor en la última repetición respecto a primera $(7,3 \pm 1,5$ vs. $6,2 \pm 1,4 \mathrm{UA})$ en un JR con una duración de 3 repeticiones de $4 \mathrm{~min}$. Atendiendo a estos resultados obtenidos parece que los JRs que se componen de repeticiones de 4 min podrían tener una mayor exigencia en la última repetición del JR. Este conocimiento podría ayudar a los cuerpos técnicos a establecer de manera más apropiada la duración total y de cada repetición de los JRs.

Cuantificar únicamente las demandas subjetivas registradas por los jugadores durante la realización de los JR no es suficiente para valorar la fatiga ocasionada, sino que también es necesario analizar el rendimiento en determinados tests físicos antes y después de cada repetición de los JRs (16). En la literatura científica no se han encontrado resultados coincidentes que permitan proporcionar una conclusión clara acerca de cómo afectan los distintos formatos de JRs en el rendimiento en el test de esprint. Mientras Rebelo et al. (17) mostraron que los jugadores empleaban más tiempo en recorrer distancias de 5 y $15 \mathrm{~m}$ después de la realización de un JR disputado en un formato de $5 \mathrm{vs5}$ (porteros incluidos) y en un Ell de $140 \mathrm{~m}^{2}$, Muñoz et al. (18), en un JR de similares características, no observaron tales diferencias en el rendimiento en distancias de $15 \mathrm{~m}$. En cambio, nuestros resultados mostraron una pérdida (muy probable - extremadamente probable pequeñas - moderadas - grandes) de rendimiento en el esprint en 10 y $30 \mathrm{~m}$ después de la última repetición en los distintos JRs respecto a antes de su comienzo. Sin embargo, diferencias poco claras y probables pequeñas fueron observadas en el rendimiento de $\mathrm{SH}$. Este es el primer estudio científico que analiza el efecto de los JRs en la capacidad de $\mathrm{SH}$ en futbolistas jóvenes. Estos resultados muestran la aparición de fatiga neuromuscular, manifestada por la pérdida de rendimiento en la capacidad de acelerar y esprintar, en la última repetición de los distintos formatos de JR. Por ello, estos resultados podrían ser útiles para los preparadores físicos de cara a planificar de manera eficaz la duración de las repeticiones y de los descansos de las tareas de entrenamiento.

En este estudio se encontraron asociaciones poco claras entre el porcentaje de cambio antes y después de la realización de los JRs en los test de $\mathrm{SH}$ y esprint en distancias de 10 y $30 \mathrm{~m}$ con la $\mathrm{Cl}$ EP total acumulada. Atendiendo a estas asociaciones y a los resultados reportados por otros autores (18) parece que la $\mathrm{CI}$ EP no es un indicador fiable para valorar la fatiga neuromuscular ocasionada a lo largo de un JR. Probablemente, y dado que también en otros estudios se ha observado que la fatiga producida en los formatos de JRs pueda tener un com- 
ponente neuromuscular, debido especialmente al importante número cambios de direcciones, aceleraciones y desaceleraciones que se producen (32), en futuras investigaciones sería interesante analizar la asociación entre el número de acciones cortas y de alta intensidad en los JRs y la pérdida de rendimiento físico en determinados tests.

\section{CONCLUSIONES}

Los diferentes formatos de JRs analizados provocaron una mayor CI EP en la última repetición respecto a la primera independientemente de las dimensiones del espacio de juego y la duración de las repeticiones. Este conocimiento podría ayudar a los cuerpos técnicos a establecer de manera más apropiada la duración total y de cada repetición de los JRs. Así mismo, mientras diferencias poco claras y probables pequeñas fueron observadas en el rendimiento de $\mathrm{SH}$ a lo largo de las repeticiones de los distintos JRs, se demostró una pérdida (muy probable extremadamente probable pequeñas - moderadas - grandes) de rendimiento en el esprint en 10 y $30 \mathrm{~m}$ después de la última repetición en los distintos JRs respecto a antes del comienzo de estos. Estos resultados muestran la aparición de fatiga neuromuscular, manifestada por la pérdida de rendimiento en la capacidad de acelerar y esprintar, en la última repetición de los distintos formatos de JR.

\section{REFERENCIAS}

1. San Roman-Quintana J, Casamichana D, Castellano J, Calleja-Gonzalez J, Jukic I, Ostojic SM. The influence of ball-touches number on physical and physiological demands of large-sided games. Kinesiology. 2013;45(2):171-8.

2. Los Arcos A, Vázquez JS, Martín J, Lerga J, Sánchez F, Villagra F, et al. Effects of small-sided games vs. interval training in aerobic fitness and physical enjoyment in young elite soccer players. PLoS One. 2015;10(9):e0137224.

3. Castellano J, Casamichana D. El arte de planificar en fútbol. $1^{\text {a }}$ Edición. Madrid: FDL; 2016.

4. Casamichana D, San Román J, Calleja-Gonzalez J, Castellano J. Los juegos reducidos en el entrenamiento en fútbol. 1 1 Edición. Madrid: FDL; 2015.

5. Casamichana D, Castellano J, Dellal A. Influence of different training regimes on physical and physiological demands during small-sided soccer games. J Strength Cond Res. 2013;27(3):690-7.

6. Castellano J, Blanco-Villaseñor A, Álvarez D. Contextual variables and time-motion analysis in soccer. Int J Sports Med. 2011;32(06):415-21.

7. Zurutuza U, Castellano J, Echeazarra I, Casamichana D. Absolute and relative training load and its relation to fatigue in football. Front Psychol. 2017;8.

8. Halouani J, Chtourou H, Gabbett TJ, Chaouachi A, Chamari K. Small-sided games in team sports training: a brief review. J Strength Cond Res. 2014;28(12):3594618. 
9. Hill-Haas S V., Dawson BT, Impellizzeri FM, Coutts AJ. Physiology of small-sided games training in football: A systematic review. Sport Med. 2011;41(3):199-220.

10. Aguiar M V, Botelho GM, Goncalves BS, Sampaio JE. Physiological responses and activity profiles of football small-sided games. J Strength Cond Res.

2013;27(5):1287-94.

11. Selmi O, Gonçalves B, Ouergui I, Sampaio J, Bouassida A. Influence of well-being variables and recovery state in physical enjoyment of professional soccer players during small-sided games. Res Sport Med. 2018;00(00):1-12.

12. Casamichana D, Castellano J. Time-motion, heart rate, perceptual and motor behaviour demands in small-sides soccer games: Effects of pitch size. J Sports Sci. 2010;28(14):1615-23.

13. Rampinini E, Impellizzeri FM, Castagna C, Abt G, Chamari K, Sassi A, et al. Factors influencing physiological responses to small-sided soccer games. J Sports Sci. 2007;25(6):659-66.

14. Fanchini M, Azzalin A, Castagna C, Schena F, Mccall A, Impellizzeri FM. Effect of Bout Duration on Exercise Intensity and Technical Performance of Small-Sided Games in Soccer. J Strength Cond Res. 2011;25(2):453-8.

15. Los Arcos A, Mendez-Villanueva A, Martínez-Santos R. In-season training periodization of professional soccer players. Biol Sport. 2017;34(2):149-55.

16. Castillo D, Yanci J, Cámara J, Weston M. The influence of soccer match play on physiological and physical performance measures in soccer referees and assistant referees. J Sport Sci. 2016;34(6):557-63.

17. Rebelo AN, Silva P, Rago V, Barreira D, Krustrup P. Differences in strength and speed demands between 4v4 and 8v8 small-sided football games. J Sport Sci. 2016;34:2246-54.

18. Muñoz J, Castillo D, Yanci J. Análisis de la percepción subjetiva del esfuerzo diferenciado y de la fatiga en distintos formatos de juegos reducidos en fútbol. Revista Iberoamericana de Ciencias la Actividad Física y el Deport. 2018;7(2):1428.

19. Sparkes W, Turner A, Weston M, Russell M, Johnston M, Kilduff L. The neuromuscular, biochemical, endocrine and mood responses to small-sided games training in professional soccer. J Strength Cond Res. 2018; 32(9): 2569-2576

20. Nevado F, Tejero CM, Paredes V, Campo J del. Análisis comparativo de las demandas físicas de dos tareas de juego reducido en fútbol profesional. Arch Med del Deport. Rev la Fed Española Med del Deport y la Confed Iberoam Med del Deport. 2015;32(2):82-6.

21. Los Arcos A, Yanci J, Mendiguchia J, Salinero JJ, Brughelli M, Castagna C. Short-term training effects of vertically and horizontally oriented exercises on neuromuscular performance in professional soccer players. Int J Sport Physiol Perform. 2014;9(3):480-8.

22. Yanci J, Los Arcos A, Camara J, Castillo D, García A, Castagna C. Effects of horizontal plyometric training volume on soccer players' performance. Res Sport Med. 2016;24(4):308-19.

23. Harriss DJ, Atkinson G. Ethical standards in sport and exercise science research: 2014 update. Int J Sports Med. 2013;34(12):1025-8. 
24. Nakamura FY, Sanchez-Sanchez J, Ramirez-Campillo R, Petisco C, GonzaloSkok O, Rodriguez-Fernandez A, et al. Effects of repeated-sprints with changes of direction on youth soccer player's performance. J Strength Cond Res. 2017; [Epub ahead of print]

25. Castellano J, Puente A, Echeazarra I, Casamichana D. Influence of the number of players and the relative pitch area per player on heart rate and physical demands in youth soccer. J Strength Cond Res. 2015;29(6):1683-91.

26. Maulder P, Cronin JB. Horizontal and vertical jump assessment: reliability, symmetry, discriminative and predictive ability. Phys Ther Sport. 2005;6(2):74-82.

27. Foster C, Florhaug JA, Franklin J, Gottschall L, Hrovatin LA, Parker S, et al. A new approach to monitoring exercise training. J strength Cond Res. 2001;15(1):109-15.

28. Hopkins WG, Marshall SW, Batterham AM, Hanin J. Progressive statistics for studies in sports medicine and exercise science. Med Sci Sports Exerc. 2009;41(1):3-13.

29. Sanchez-Sanchez J, Hernández D, Casamichana D, Martínez-Salazar C, RamirezCampillo R, Sampaio J. Heart rate, technical performance, and session-rpe in elite youth soccer small-sided games played with wildcard players. J Strength Cond Res. 2017;31(10):2678-85.

30. Buchheit M, Lacome M, Cholley Y, Simpson BM. Neuromuscular responses to conditioned soccer sessions assessed via gps-embedded accelerometers: insights into tactical periodization. Int J Sports Physiol Perform. 2018;13:577-83.

31. Lacome M, Simpson BM, Cholley Y, Lambert P, Buchheit M. Small-sided games in elite soccer: does one size fit all? Int J Sports Physiol Perform. 2018;13(5):568-76.

32. Gaudino P, laia FM, Strudwick AJ, Hawkins RD, Alberti G, Atkinson G, et al. Factors influencing perception of effort (session rating of perceived exertion) during elite soccer training. Int J Sport Physiol Perform. 2015;10(7):860-4. 